\title{
Structural brain changes in patients with Huntington's disease participating in a randomized, double-blind, placebo-controlled trial of ethyl-eicosapentaenoic acid
}

\author{
Basant K Puri ${ }^{1 *}$, Graeme M Bydder ${ }^{2}$, Mehar S Manku ${ }^{3}$, Anthony Clarke ${ }^{3}$, Christian F Beckmann ${ }^{4}$ \\ From $1^{\text {st }}$ International Congress on Neurobiology and Clinical Psychopharmacology \\ and European Psychiatric Association Conference on Treatment Guidance \\ Thessaloniki, Greece. 19-22 November 2009
}

\section{Background}

Ultra-pure ethyl-eicosapentaenoic acid (ethyl-EPA) is a semi-synthetic, ethyl ester of the long-chain fatty acid eicosapentaenoic acid which has been shown to be associated with clinical improvement in motor functioning in Huntington's disease. The aim was to determine the extent to which it might reduce the rate of progress of cerebral atrophy.

\section{Materials and methods}

High-resolution MRI cerebral scanning was carried out at baseline, six months and one year in 30 patients with stage I or II Huntington's disease who took part in a randomized, double-blind, placebo-controlled trial of $2 \mathrm{~g}$ daily ethyl-EPA or liquid paraffin, using a $1.0 \mathrm{~T}$ Picker HPQ scanner. For each subject and each pair of T1 images, the two-timepoint percentage brain volume change was estimated in a double-blind fashion using SIENA (Structural Image Evaluation, using Normalisation, of Atrophy), Version 2.5, part of the FSL (version 4.0) comprehensive library of analysis tools.

\section{Results}

Overall, patients treated with ethyl-EPA had a reduced mean rate of atrophy in all comparisons compared with the placebo group. There was no significant effect of age at the time of scanning on these results. Areas of significant group-level reduction in brain atrophy between patients receiving ethyl-EPA and those receiving placebo were found. Significant changes were observed at the head of the caudate nucleus and the posterior thalamus.

\section{Conclusions}

Treatment with ethyl-EPA is associated with significant reduction in brain atrophy in Huntington's disease, particularly in the head of the caudate and the posterior thalamus. No other drug tested in HD has shown this effect.

\section{Acknowledgements}

We thank the MRC and Amarin Neuroscience.

\section{Author details}

${ }^{1}$ Imaging Sciences Department, Hammersmith Hospital, Imperial College London, UK. ²Department of Radiology, University of California, San Diego, School of Medicine, San Diego, CA, USA. ${ }^{3}$ Amarin Neuroscience Limited, The Oxford Science Park, Oxford, UK. ${ }^{4}$ Clinical Neuroscience Department, Imperial College London and FMRIB Centre University of Oxford, John Radcliffe Hospital, Oxford, UK.

Published: 22 April 2010

doi:10.1186/1744-859X-9-S1-S151

Cite this article as: Puri et al:: Structural brain changes in patients with Huntington's disease participating in a randomized, double-blind, placebo-controlled trial of ethyl-eicosapentaenoic acid. Annals of General Psychiatry 2010 9(Suppl 1):S151. 